\title{
Gene expression profiling associated with the progression to poorly differentiated thyroid carcinomas
}

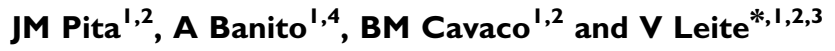 \\ 'Centro de Investigação de Patobiologia Molecular (CIPM), Instituto Português de Oncologia de Lisboa Francisco Gentil, Lisboa I099-023, Portugal; \\ ${ }^{2}$ Centro de Estudos de Doenças Crónicas (CEDOC), Faculdade de Ciências Médicas da Universidade Nova de Lisboa, Lisboa II 69-056, Portugal; \\ ${ }^{3}$ Serviço de Endocrinologia, Instituto Português de Oncologia de Lisboa Francisco Gentil, Lisboa 1099-023, Portugal
}

BACKGROUND: Poorly differentiated thyroid carcinomas (PDTC) represent a heterogeneous, aggressive entity, presenting features that suggest a progression from well-differentiated carcinomas. To elucidate the mechanisms underlying such progression and identify novel therapeutic targets, we assessed the genome-wide expression in normal and tumour thyroid tissues.

METHODS: Microarray analyses of 24 thyroid carcinomas -7 classic papillary, 8 follicular variants of papillary (fvPTC), 4 follicular (FTC) and 5 PDTC - were performed and correlated with RAS, BRAF, RET/PTC and PAX8-PPARG alterations. Selected genes were validated by quantitative RT-PCR in an independent set of 28 thyroid tumours.

RESULTS: Unsupervised analyses showed that gene expression similarity was higher between PDTC and fvPTC, particularly for tumours harbouring RAS mutations. Poorly differentiated thyroid carcinomas presented molecular signatures related to cell proliferation, poor prognosis, spindle assembly checkpoint and cell adhesion. Compared with normal tissues, PTC had 307 out of 494 (60\%) genes over-expressed, FTC had I 37 out of I7I (80\%) genes under-expressed, whereas PDTC had 92 out of I07 (86\%) genes under-expressed, suggesting that gene downregulation is involved in tumour dedifferentiation. Significant UHRFI and ITIH5 deregulated gene expression in PDTC, relatively to normal tissues, was confirmed by quantitative RT-PCR.

CONCLUSION: Our findings suggest that fvPTC are possible precursors of PDTC. Furthermore, UHRFI and ITIH5 have a potential therapeutic/prognostic value for aggressive thyroid tumours.

British Journal of Cancer (2009) I I I, |782- |79I. doi:I0.1038/sj.bjc.6605340 www.bjcancer.com

Published online 6 October 2009

(C) 2009 Cancer Research UK

Keywords: dedifferentiation; genome-wide expression; oligonucleotide microarray; poorly differentiated thyroid carcinoma; molecular signatures

Most thyroid neoplasias derive from follicular cells and show a wide range of biological behaviours from indolent to highly invasive cancers (DeLellis et al, 2004). Well-differentiated thyroid cancers (WDTC), such as papillary thyroid carcinoma (PTC) and follicular thyroid carcinoma (FTC), are usually treated successfully with surgery and radioactive iodine; however, poorly differentiated thyroid carcinoma (PDTC) and anaplastic (or undifferentiated) thyroid carcinoma (ATC) can behave aggressively with no effective form of treatment (Patel and Shaha, 2006).

Previous reports suggest a model of progression from WDTC to PDTC and to ATC. PDTC show limited follicular cell differentiation and are, both morphologically and behaviourally, positioned between well- and undifferentiated carcinomas (DeLellis et al, 2004). Indeed, cases of WDTC containing areas of pooror undifferentiation, as well as, cases of PDTC/ATC containing

*Correspondence: Dr V Leite; E-mail: vleite@ipolisboa.min-saude.pt

${ }^{4}$ Current address: Cell Proliferation Group, MRC Clinical Sciences Centre, Faculty of Medicine, Imperial College, Hammersmith Hospital Campus, WI2 ONN London, UK

Received 7 April 2009; revised 2 September 2009; accepted 4 September 2009; published online 6 October 2009 well-differentiated areas, have been widely detected (Lam et al, 2000). Progression is further suggested by the sequential increase in chromosomal abnormalities from WDTC to PDTC and ATC (Wreesmann et al, 2002; Rodrigues et al, 2004). Mutations in the RAS and BRAF genes also support a model of tumour progression, as the frequency of these events in PDTC is midway between well-differentiated and undifferentiated carcinomas, rather than being randomly distributed (Garcia-Rostan et al, 2003; Nikiforova et al, 2003). Other alterations, such as tumour suppressor TP53 mutations, are specifically found in PDTC and ATC, and are often associated with RAS or BRAF mutations (Quiros et al, 2005; Wang et al, 2007), suggesting an accumulation of events during progression. Nevertheless, it is not clear whether PDTC derive from either PTC or FTC, or whether they arise de novo. In addition, the genetic and epigenetic mechanisms underlying the process remain ill defined.

Genome-wide expression analysis has been successfully used to identify molecular signatures, improving the diagnosis and prognosis of several types of tumours (Quackenbush, 2006). For thyroid neoplasias, one of the earliest reports of genomewide expression analysis described a consistent gene expression profile that distinguished PTC from normal cells 
(Huang et al, 2001). Most of the genes identified in this work were corroborated in subsequent studies. Gene expression studies have also been used to differentiate benign from malignant thyroid tumours, and correlate gene expression patterns with specific mutations or rearrangements in PTC and FTC (for review, see Eszlinger et al, 2007).

To our knowledge, only two studies have addressed the genomewide expression of PDTC. One of these studies compared gene expression of PDTC and ATC cell lines to normal thyroid tissue, and showed that these cells presented largely altered expression profiles that have been associated with the cancer process (Rodrigues et al, 2007). Although the authors confirmed some of the abnormal expressed genes in primary tumours, it has been shown that immortal cell lines may not fully reflect the functional aspects of the tumours, and that some molecular processes might be specifically acquired during the immortalisation step (Dairkee et al, 2004). In the other study, which used WDTC, PDTC and ATC primary tumours, deregulation of different molecular pathways, such as the MAPK signalling pathway, focal adhesion and cell motility, cell proliferation and cell-cycle progression, was associated with dedifferentiation in PDTC and ATC (Montero-Conde et al, 2008).

In this study, we used the array platform GeneChip Human Genome U133 Plus 2.0 (HG-U133 Plus 2.0) to analyse the expression of a wide range of genes $(>30000)$ in well- and poorly differentiated thyroid tumours and, to correlate, for the first time, gene expression with BRAF, RAS, RET/PTC and PAX8-PPARG alterations.

\section{MATERIALS AND METHODS}

\section{Tissue samples}

Both tumour and normal thyroid tissue samples were obtained at time of surgery, and were immediately frozen in liquid nitrogen. Histological classifications followed the criteria described in World Health Organization (WHO) classification of thyroid tumours (DeLellis et al, 2004). All samples were obtained with permission, and the project was approved by our institution ethical committee.

The microarray sample set consisted of a total of 24 tumour samples - 5 PDTC, 7 classic PTC (cPTC), 8 follicular variants of PTC (fvPTC) and 4 FTC (Supplementary Table 1). A pool of human thyroid total RNA obtained from 65 Caucasian individuals with 18-61-years old, whom died from sudden death (BD Bioscience, Franklin Lakes, NJ, USA), and 2 normal tissue samples taken from the opposite lobe of thyroid tumours, were also processed.

An independent sample set, consisting of five PDTC, seven cPTC, seven fvPTC, nine FTC and six normal thyroid tissues taken from the opposite lobe of thyroid tumours, was used for quantitative real-time RT-PCR. Expression was also studied in two poorly differentiated thyroid cancer cell lines, T243 and T351 (described earlier by Rodrigues et al, 2007), kindly supplied by Dr Lúcia Roque, from Centro de Investigação de Patobiologia Molecular (CIPM), Instituto Português de Oncologia de Lisboa Francisco Gentil, Lisbon, Portugal.

\section{Total RNA isolation/extraction}

Total RNA was extracted and purified using the RNeasy Mini kit (Quiagen, Hamburg, GmbH, Germany) according to the manufacturer's protocol, and quantified by UV spectrophotometry (NanoDrop ND-1000, Thermo Fisher Scientific, Wilmington, DE, USA). RNA integrity was assessed by micro capillary electrophoresis (Agilent 2100 Bioanalyzer, Santa Clara, CA, USA) and samples with RNA Integrity Number equal or higher than 7.7 were selected for microarray analysis.

\section{RNA processing and hybridisation}

RNA samples were processed following the one-cycle eukaryotic target labelling protocol from Affymetrix, and were hybridised using the HG-U133 Plus 2.0 Array (Affymetrix, Santa Clara, CA, USA). Hybridisation results were scanned using the GeneChip Scanner 3000 and stored in the GeneChip Operating Software.

\section{Microarray data analysis}

Partek Genomics Suite Software (Partek Inc, St Louis, MO, USA) was used for unsupervised analyses. First, array data were normalised and the expression levels were determined applying the robust multi-array average method (Irizarry et al, 2003), and data were corrected for non-biological factors. Samples were represented three dimensionally, according to the expression levels of all probe sets, by principal components analysis (PCA). Probesets data were also used to obtain a dendogram of the samples, by hierarchical clustering, with the Pearson correlation coefficient.

DNA-ChipAnalyzer (dChip) 2006 software (Li and Wong, 2001) was used to obtain differentially expressed genes between groups. Arrays were normalised with the invariant set normalisation method and expression levels were calculated by model-based expression analysis with perfect match-only model. Probe sets that were absent in all samples or those that did not change across samples (coefficient of variation lower than 0.2 and higher than 10) were eliminated from further analysis. Probe sets were considered to be differentially expressed between two groups when the lower $90 \%$ limit of the confidence interval of the fold change (ratio of the expression level in the two groups) was equal or higher than twofold, with an unpaired $t$-test considered significant at $P \leqslant 0.01$. Onto-Express from Onto-Tools package (Khatri et al, 2002) was used to classify genes differentially expressed according to their biological role.

Gene set enrichment analysis (GSEA) software (Subramanian et al, 2005) was used to determine whether members of defined groups of genes, which share common features (gene sets), are preferentially placed towards the top or the bottom of a list of genes. In this list, genes were ranked according to the differential expression between two sample groups. This method was applied using two catalogues of gene sets: one whose products are involved in specific pathways/functions and another defined by expression neighbourhoods, which indicates molecular signatures associated with cancer-related genes. Statistical significance was estimated by a nominal $P$ value obtained by phenotype permutation. $P$ values were corrected for multiple hypothesis testing using false discovery rate and family wise-error rate (FWER). Gene sets were considered significant at $P<0.05$ and with FWER $\leqslant 0.25$.

\section{First-strand cDNA synthesis}

cDNA was synthesised from $1 \mu \mathrm{g}$ of total RNA (for cDNA sequence analysis) or $2 \mu \mathrm{g}$ of total RNA (for quantitative RT-PCR), at $37^{\circ} \mathrm{C}$ for $90 \mathrm{~min}$, using random primer $\mathrm{p}(\mathrm{dN})_{6}$ (Roche Diagnostics Corporation, Indianapolis, IN, USA) and SuperScript II reverse transcriptase (Invitrogen, Paisley, UK).

\section{Mutational analysis of the RAS, BRAF genes and PAX8-PPARG, RET/PTC rearrangements}

Mutational analysis was undertaken using CDNA from the tumour samples of the microarray set. PTC were screened for $B R A F$ mutations and rearrangements of RET/PTC and, in addition, follicular variants were also analysed for $R A S$ mutations and $P A X 8-P P A R G$ rearrangements. FTC were screened for RAS and $P A X 8-P P A R G$ rearrangements. PDTC were analysed for $B R A F, R A S$ and $P A X 8-P P A R G$ genes. Primers were designed to amplify exon 
15 of the BRAF gene, exons 2 and 3 of the $N-$, KRAS genes and exons 1 and 2 of the HRAS gene. Primers flanking the respective fusion points were used to screen the presence of $R E T / P T C 1$, RET/PTC2, RET/PTC3 and PAX8-PPARG fusion transcripts, as described earlier (Marques et al, 2002; Rebelo et al, 2003). Sequencing analysis, to search for mutations and to confirm the rearrangements, was performed with the same primers as for PCR, using the Big Dye Terminator v1.1 Cycle Sequencing kit (Applied Biosystems, Foster City, CA, USA), according to the manufacturer's protocol. Sequencing products were separated in an ABI Prism 310 Genetic Analyser (Applied Biosystems) and analysed with the Sequence Analysis Software version 3.4.1 (Applied Biosystems). Primer sequences and assay conditions are available on request.

\section{Quantitative real-time RT - PCR}

Real-time RT-PCR assays were performed in 96-well reaction plates (MicroAmp Optical 96-Well Reaction Plate; Applied Biosystems) on an ABI Prism 7900 HT Sequence Detection System (Applied Biosystems) with the SDS Software version 2.3 (Applied Biosystems). PCR amplifications were performed using for each gene, pre-developed primers and probe (Inventoried TaqMan Gene Expression Assays ID: Hs00218544_m1 (PBK); Hs00273589_m1 (UHRF1); Hs00228960_m1 (ITIH5); Applied Biosystems), and TaqMan Universal PCR Master Mix (Applied Biosystems), according to the manufacturer's protocol. To normalise differences in the amount of template used, glyceraldehyde-3-phosphate dehydrogenase (GAPDH) transcript was used as an endogenous control (Pre-Developed TaqMan Assay Reagents Human GAPDH; Applied Biosystems). Two-fold serial dilutions were used to apply the relative standard curve method. A pool of five normal thyroid tissues was used as calibrator to determine the relative expression in samples. All reactions, including a control without template, were performed in triplicate.

Quantitative RT - PCR results were analysed using the GraphPad Prism version 4.00 (GraphPad Software, Inc). Intensity levels were calculated as mean \pm standard error of the mean (s.e.m.). Comparisons between sample groups were performed using the Kruskal-Wallis with Dunn's Multiple Comparison test, because samples distribution was not Gaussian or variances between groups were not equal. Correlations of quantitative RT - PCR data with other variables were performed using the Pearson or the Spearman correlations (for a non-Gaussian distribution). The correlations and differences between group means were considered significant at $P<0.05$.

\section{RESULTS}

\section{Mutation screening}

Tumour samples were screened for mutations in MAPK pathway effectors, which are frequently mutated in thyroid cancer (Supplementary Table 1). The BRAF V600E substitution was only present in CPTC, accounting for $57.1 \%$ (four out of seven) of the cases. On the other hand, mutations of $N$ - or KRAS genes were observed in $50 \%$ (four out of eight) of the fvPTC and in $40 \%$ (two out of five) of PDTC. All FTC $(n=4)$ were RAS negative. The $P A X 8-P P A R G$ fusion gene was found in $12.5 \%$ (one out of eight) of fvPTC and in $25 \%$ (one out of four) of FTC. No RET/PTC1, -2 or -3 rearrangements were identified in PTC. However, as other rearrangements involving the RET (Ciampi et al, 2007) or NTRK1 (Pierotti and Greco, 2006) genes have also been described in PTC, we specifically analysed RET and NTRK1 microarray mRNA expression in PTC negative for mutations. In one cPTC (sample 2 - Supplementary Table 1), a 20 -fold increase in RET expression was detected in comparison to the other samples. FISH confirmed the presence of a RET/PTC rearrangement in $37 \%$ (71 out of 194) of these tumour cells (data not shown).

\section{Unsupervised analyses for global gene expression profiling}

We carried out unsupervised analyses to examine the relationship between gene expression, tumour histotype and mutational status. Global gene expression similarity between the 27 samples was examined using hierarchical clustering (Figure 1). As represented on the dendrogram, distinct profiles separated FTC from the other tumours. Interestingly, a case of fvPTC diffuse (or multinodular) with a $P A X 8-P P A R G$ rearrangement clustered with FTC. Different molecular signatures were present in the PTC sub-set: cPTC formed a separate sub-group from fvPTC and, among these PTC subtypes, samples with $R A S / B R A F$ mutations were separated from samples without mutations. By PCA of data for all probe sets, which represents the samples three dimensionally according to the global gene expression profile, the FTC and the fvPTC diffuse (or multinodular) also formed a group apart from the other tumours and normal thyroid samples, which tended to cluster together (Supplementary Figure 1; Supplementary Movie 1). PDTC samples, particularly those with RAS mutations, clustered with PTC in both representations.

\section{Genes differentially expressed between tumours and normal tissue}

Differentially expressed genes were defined as those with an expression level equal, or higher, than two-fold in a group relatively to another, with a $P$ value $\leqslant 0.01$. We compared each

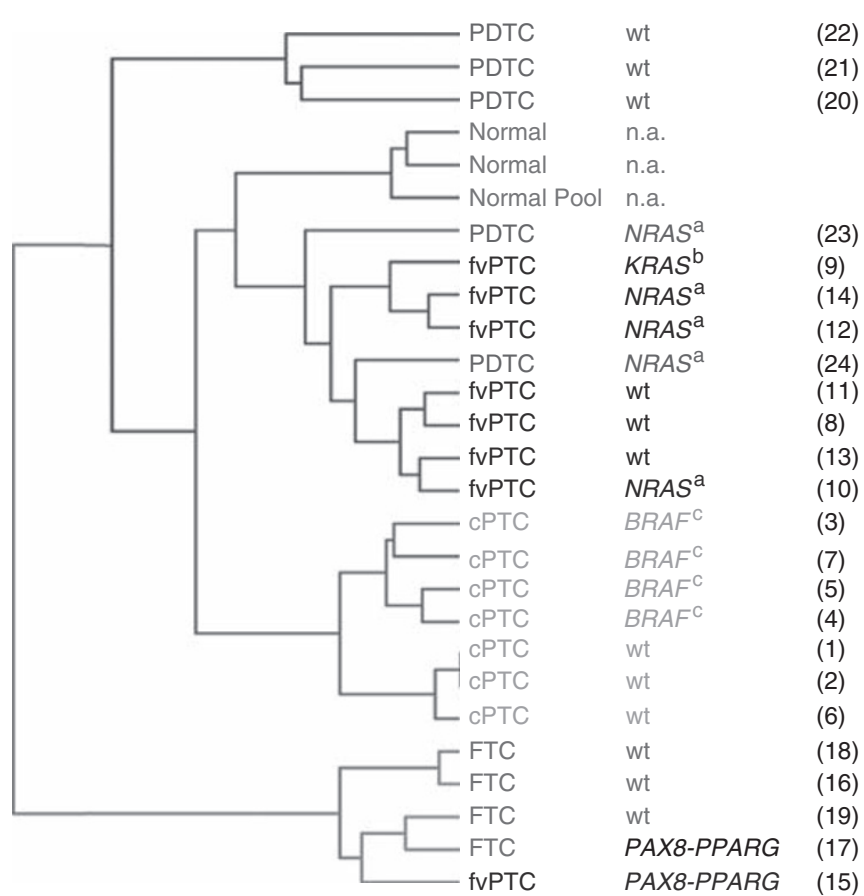

Figure I Global gene expression similarity between samples using the unsupervised hierarchical clustering method. In the dendogram, distance separating samples represents the gene expression resemblance between them. The result of mutational analysis for each tumour specimen is shown. Wild-type (wt) label denotes absence of mutation in screened genes; ${ }^{\mathrm{a}} \mathrm{Q} 6$ IR mutant of NRAS; ${ }^{\mathrm{b}} \mathrm{G}$ I 3R mutant of KRAS; ' $\mathrm{V} 600 \mathrm{E}$ mutant of BRAF; in parenthesis is indicated the sample number assigned in Supplementary Table I. cPTC = classic papillary thyroid carcinoma; FTC = follicular thyroid carcinoma; $\mathrm{FVPTC}=$ follicular variant of papillary thyroid carcinoma; PDTC $=$ poorly differentiated thyroid carcinoma; n.a. $=$ not applicable. 
tumour group (cPTC, fvPTC, FTC and PDTC) with the three normal thyroid samples, and we found over-expression of about $60 \%$ of probe sets for both CPTC and fvPTC, whereas in FTC and PDTC, about $80 \%$ of the probe sets were under-expressed (Figure 2). PDTC had 92 downregulated genes relatively to normal tissues (Supplementary Table 2), but only 15 out of the 107 genes differentially expressed were over-expressed (Table 1).

The biological processes mainly represented by the probe sets differentially expressed between thyroid tumours and normal tissues were the signal transduction, cell adhesion, regulation of transcription and cell proliferation/cell cycle (Supplementary Figure 2). We were also able to identify 11 probe sets that were under-expressed in all tumours comparatively to normal tissues (Table 2).

\section{Genes specific for PDTC}

The PDTC group had 3, 8 and 1 over-expressed probe sets and 11, 154 and 59 under-expressed probe sets compared with FTC, cPTC and fvPTC, respectively (data not shown). Only two probe sets were consistently under-expressed in the PDTC tumour set comparatively to the WDTC (Table 1).

\section{GSEA for PDTC}

GSEA is another method for interpreting gene expression data that focus on groups of genes sharing common biological function, chromosomal location or regulation. This approach can show important effects on pathways, which might be missed in singlegene analysis (Subramanian et al, 2005). We applied this methodology to identify pathways altered in thyroid tumour progression. There were no statistically significant functionaldefined gene sets enriched in PDTC samples vs WDTC, still we analysed the 20 most relevant results (Table 3 ). The budding uninhibited by benzimidazoles 1 homolog (yeast) (BUB1) was the most represented gene being present in 13 of the 20 gene sets. Cell division cycle 2, G1-S and G2-M (CDC2) and Cyclin B2 (CCNB2) were represented in 12 of the 20 gene sets, and 10 gene sets contained MAD2 mitotic arrest-deficient-like 1 (yeast) (MAD2L1), topoisomerase (DNA) II $\alpha 170 \mathrm{kDa}$ (TOP2A), cyclin-dependent kinase inhibitor 3 (CDK2-associated dual specificity phosphatase) $(C D K N 3)$ and centromere protein $\mathrm{A}$ (CENPA). In addition, statistically significant molecular signatures of four deregulated genes, which are involved in the cancer process, were identified in PDTC (Table 3 - Expression neighbourhoods-defined gene sets).

\section{Validation of microarray gene expression}

Real-Time RT-PCR was performed to validate three genes differentially expressed between PDTC and normal thyroid samples: ubiquitin-like, containing $\mathrm{PHD}$ and RING finger domains, 1 (UHRF1), PDZ-binding kinase $(P B K)$ and inter-alpha (globulin) inhibitor H5 (ITIH5). This validation was processed in an independent sample set of 28 tumours (Figure 3). UHRF1 and $P B K$ had increased expression in all tumour samples relatively to normal tissue samples, but the highest expression levels were detected in PDTC. Differences in the UHRF1 expression (Figure 3B) between PDTC and normal tissue were statistically significant $(11.77 \pm 3.07$ vs $1.77 \pm 0.36 ; P<0.01)$, even if only considering the expression in the independent set $(14.30 \pm 5.49$ vs $1.77 \pm 0.36 ; P<0.05$ ) (Figure $3 \mathrm{~A}$ ). On the other hand, differences in the PBK expression were not statistically significant (Figures 3C and D). ITIH5 expression was decreased in all tumours samples relatively to normal tissues (Figures $3 \mathrm{E}$ and F). Statistically significant differences were detected in all tumour groups relatively to normal samples, except in cPTC (PDTC: $0.22 \pm 0.17$, $P<0.001$; FTC: $0.15 \pm 0.05, P<0.001$; fvPTC: $0.22 \pm 0.03, P<0.05 v s$ normal: $1.00 \pm 0.10)$. Differences were also significant when considering the independent set of samples (PDTC: $0.41 \pm 0.33$,
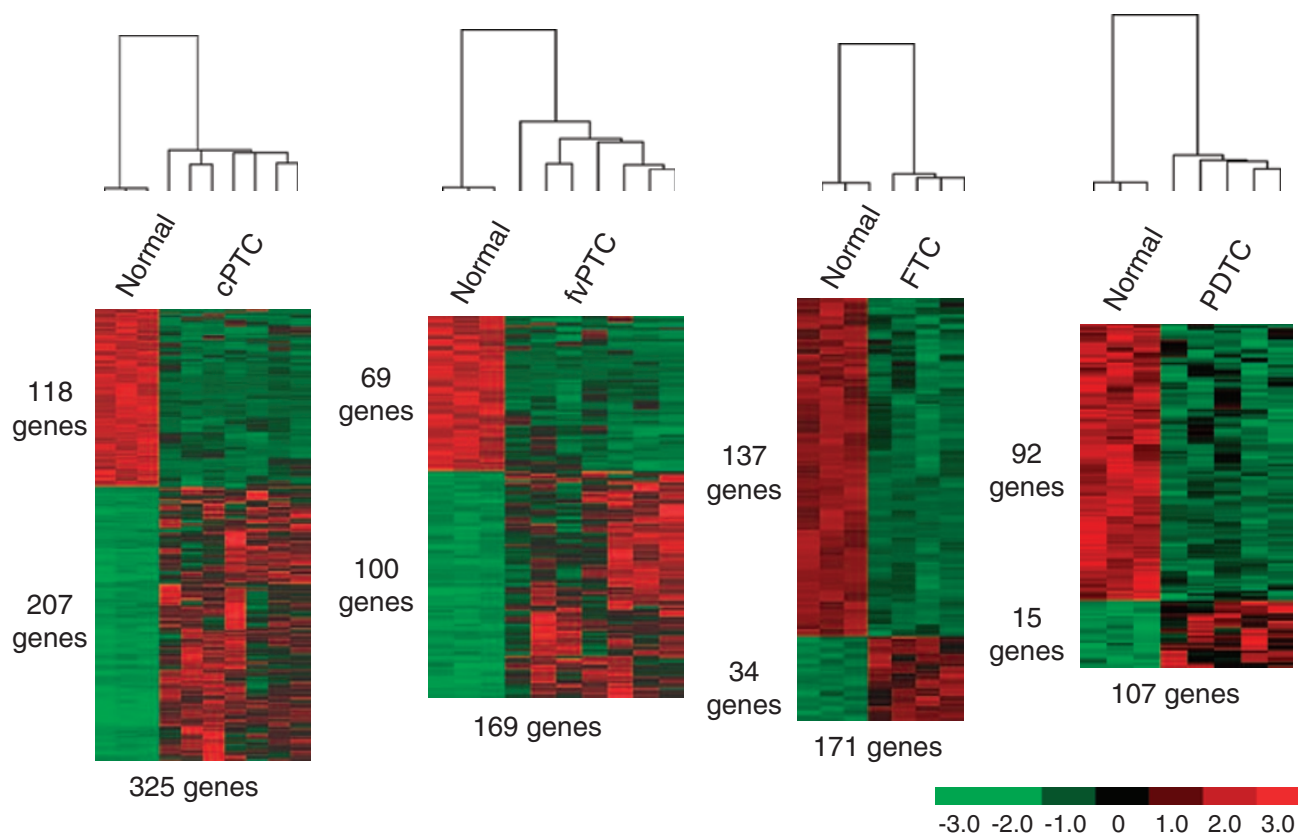

Figure 2 Expression profile of the genes differentially expressed between tumours and normal tissues. Each tumour histology was compared with the normal thyroid tissues and expression levels of the genes differentially expressed were represented. Expression levels are indicated by colour intensities in which green and red correspond, respectively, to a lower and a higher expression than the mean value for the gene, in all samples being compared. On the left of each profile, the number of under- and over-expressed genes in the tumour set is shown. At the bottom, the total number of differentially expressed genes is indicated. Only one probe set was considered for each gene. CPTC = classic papillary thyroid carcinoma; FTC = follicular thyroid carcinoma; fvPTC $=$ follicular variant of papillary thyroid carcinoma; PDTC = poorly differentiated thyroid carcinoma. 
Table I Main characteristics of differentially expressed genes in poorly differentiated tumours

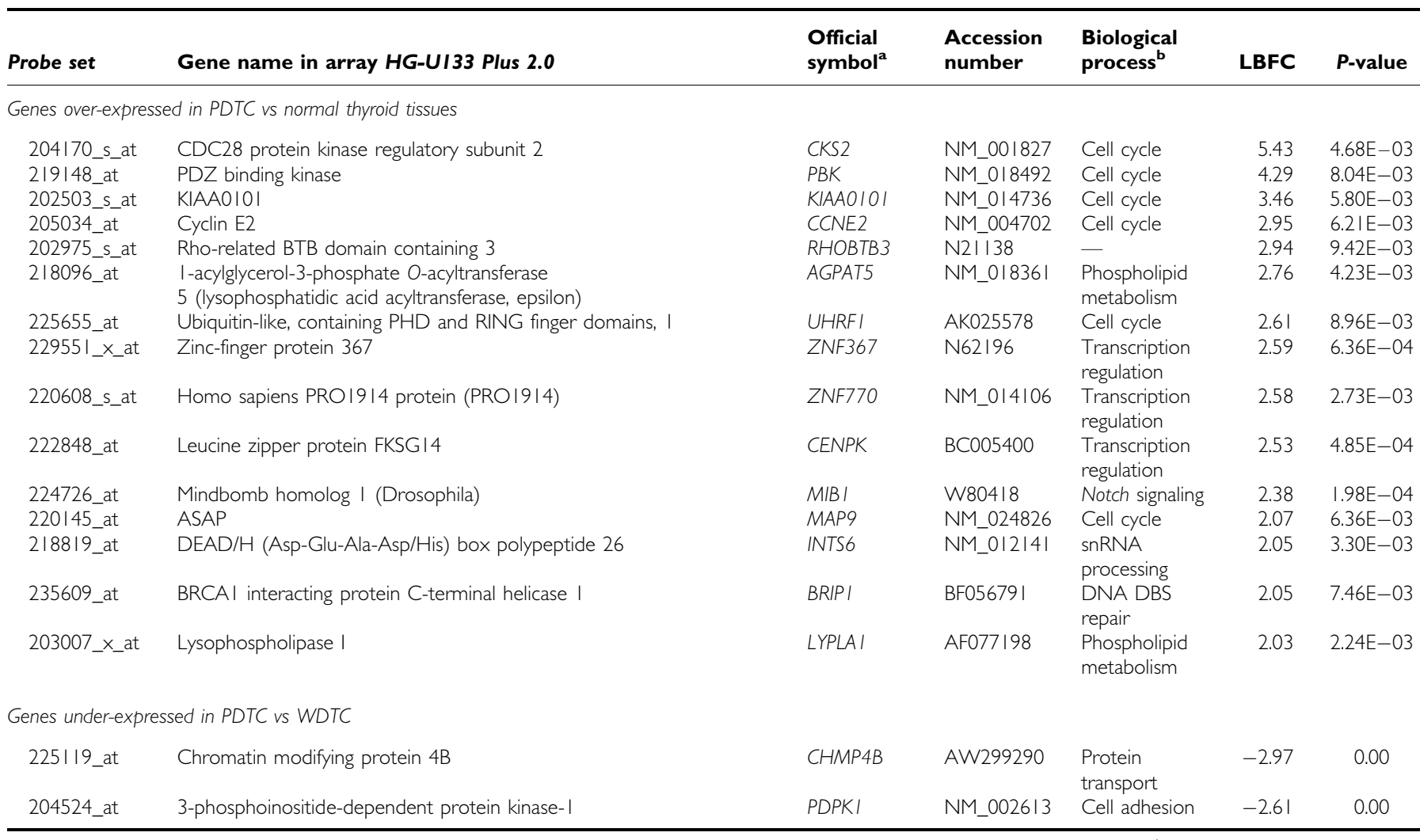

Abbreviations: DBS = double-strand breaks; $\mathrm{LBFC}=$ lower bound of fold change; snRNA = small nuclear RNA. ${ }^{2}$ Assigned in EntrezGene. ${ }^{b}$ Information taken from Online Mendelian Inheritance in Man (OMIM) or from EntrezGene. $P$ values for difference in mean expression between groups were calculated using an unpaired $t$-test.

Table 2 Main characteristics of differentially expressed genes in the four thyroid tumour histotypes vs normal thyroid tissues

\begin{tabular}{|c|c|c|c|c|c|c|}
\hline Probe set & $\begin{array}{l}\text { Gene name in array HG-UI33 } \\
\text { Plus } 2.0\end{array}$ & $\begin{array}{l}\text { Official } \\
\text { symbol }^{\mathrm{a}}\end{array}$ & $\begin{array}{l}\text { Accession } \\
\text { number }\end{array}$ & Biological process ${ }^{b}$ & LBFC & P-value \\
\hline \multicolumn{7}{|c|}{ Under-expressed genes in the four types of tumours vs normal thyroid tissues } \\
\hline $\begin{array}{l}\text { 212713_at } \\
\text { 1556427_s_at } \\
\text { 219778_at } \\
\text { 205413_at } \\
\text { 206201_s_at }\end{array}$ & $\begin{array}{l}\text { Microfibrillar-associated protein } 4 \\
\text { Similar to hypothetical protein } \\
\text { Zinc-finger protein, multitype } 2 \\
\text { Chromosome } 1 \text { I open reading frame } 8 \\
\text { Mesenchyme homeo box } 2 \text { (growth } \\
\text { arrest-specific homeo box) }\end{array}$ & $\begin{array}{l}\text { MFAP4 } \\
\text { LOC221091 } \\
\text { ZFPM2 } \\
\text { MPPED2 } \\
\text { MEOX2 }\end{array}$ & $\begin{array}{l}\text { R72286 } \\
\text { AL834319 } \\
\text { NM_0I I2082 } \\
\text { NM_00I584 } \\
\text { NM_005924 }\end{array}$ & $\begin{array}{l}\text { Cell adhesion } \\
\text { - } \\
\text { Transcription regulation } \\
\text { Nervous system development } \\
\text { Development }\end{array}$ & $\begin{array}{l}-4.20 \\
-4.07 \\
-4.03 \\
-3.59 \\
-2.92\end{array}$ & $\begin{array}{l}3.81 \mathrm{E}-04 \\
5.57 \mathrm{E}-03 \\
7.60 \mathrm{E}-03 \\
9.00 \mathrm{E}-05 \\
5.85 \mathrm{E}-03\end{array}$ \\
\hline 217525_at & Olfactomedin-like I & OLFMLI & AW305097 & Cell proliferation & -2.64 & $2.34 \mathrm{E}-04$ \\
\hline
\end{tabular}

Abbreviation: LBFC = lower bound of fold change. ${ }^{a}$ Assigned in EntrezGene. ${ }^{b}$ Information taken from Online Mendelian Inheritance in Man (OMIM) or from EntrezGene. $P$ values for difference in mean expression between tumours and normal tissues were calculated using an unpaired t-test.

$P<0.05$; FTC: $0.14 \pm 0.07, \quad P<0.001$ vs normal: $1.00 \pm 0.10)$ Additionally, we assessed UHRF1 and ITIH5 expressions in two PDTC cell lines. No significant increase in UHRF1 expression was detected (mean \pm standard deviation: $1.86 \pm 0.16$ and $1.43 \pm 0.16 v s$ $1.77 \pm 0.36)$. On the other hand, ITIH5 expression was undetectable (Ct values $>37$ ) in both cell lines. Correlation of quantitative RT-PCR data with the expression levels obtained in the microarray analysis was statistically significant (Pearson correlation $r=0.61$ for UHRF1 with $P=0.0015$; Spearman correlation $r=0.70$ for $P B K$ with $P=0.0001$; Pearson correlation $r=0.94$ for ITIH5 with $P<0.0001)$. There was no correlation of gene expression with tumour size, gender or age, except for ITIH5, whose decreased expression was associated with larger tumours (Spearman correlation $r=-0.33$ with $P=0.02$ ). 
Table 3 Gene sets enriched in the poorly differentiated vs the well-differentiated groups

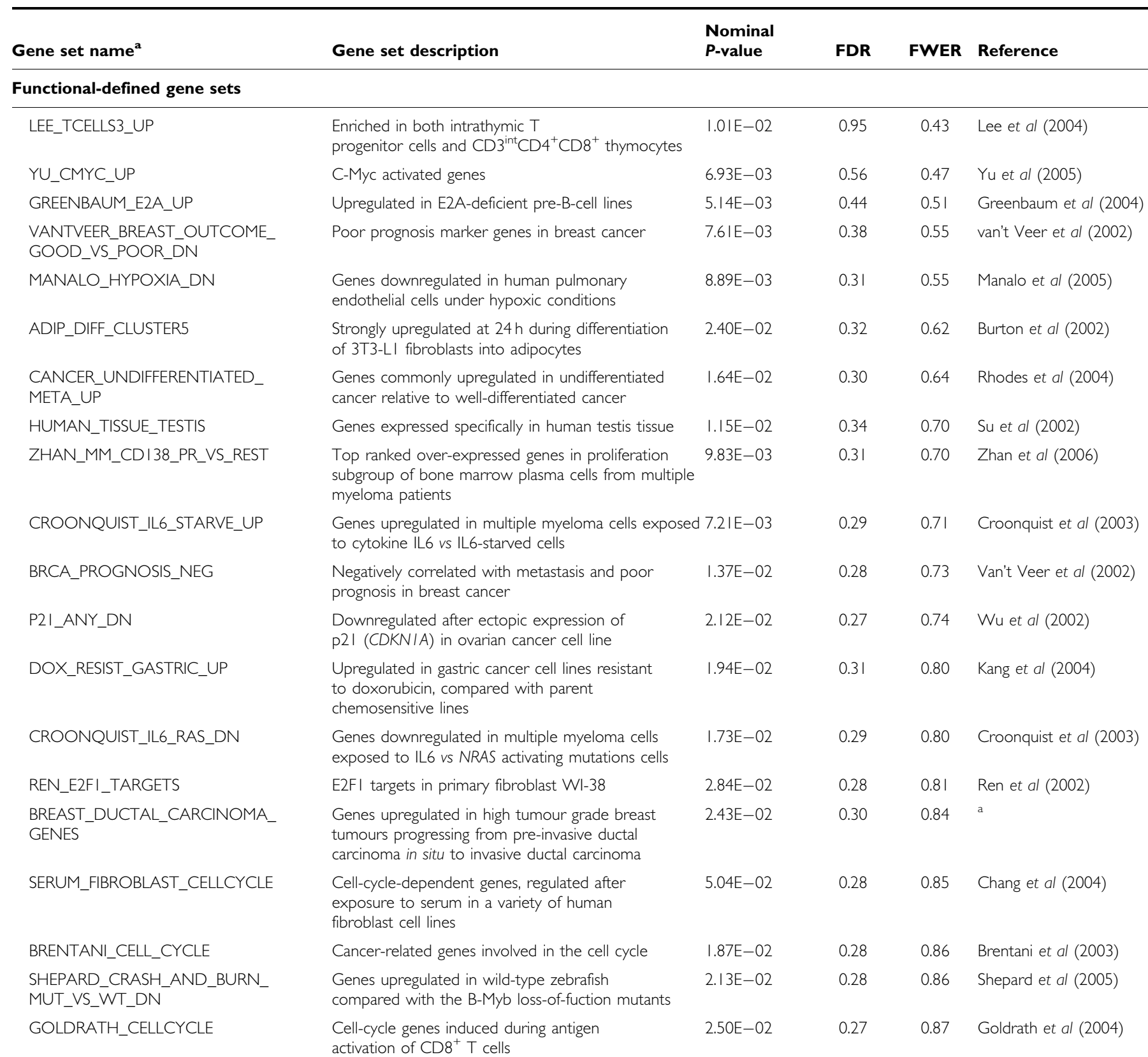

\section{Expression neighbourhoods-defined gene sets}

\begin{tabular}{|c|c|c|c|c|c|}
\hline GNF2_CKSIB & $\begin{array}{l}\text { Expression neighbourhood of CKSIB } \\
\text { in the GNF2 expression compendium }\end{array}$ & 0.00 & 0.04 & 0.02 & a \\
\hline MORF_BUBI & $\begin{array}{l}\text { Expression neighbourhood of } B U B / \text { in } \\
\text { the MORF expression compendium }\end{array}$ & 4.74E-03 & 0.14 & 0.10 & a \\
\hline GNF2_ESPLI & $\begin{array}{l}\text { Expression neighbourhood of ESPLI } \\
\text { in the GNF2 expression compendium }\end{array}$ & $9.59 \mathrm{E}-03$ & 0.18 & 0.21 & $\mathrm{a}$ \\
\hline
\end{tabular}

Abbreviations: $\mathrm{BUB} \mid=$ budding uninhibited by benzimidazoles I homolog (yeast); $\mathrm{B} \cup \mathrm{B} \mid \mathrm{B}=$ budding uninhibited by benzimidazoles $\mid$ homolog beta (yeast); $\mathrm{CD}=\mathrm{cluster}$ of differentiation; IL6= interleukin 6; CDKNIA = cyclin-dependent kinase inhibitor IA (p2I, Cip I); CKSIB =CDC28 protein kinase regulatory subunit IB; ESPLI = extra spindle pole bodies homolog I (S. cerevisiae); FDR = false discovery rate; FWER = family wise-error rate. ${ }^{\mathrm{a}}$ Assigned in Molecular Signatures Database (www.broad.mit.edu/gsea/msigdb/ index.jsp). References are supplied as supplementary data. 

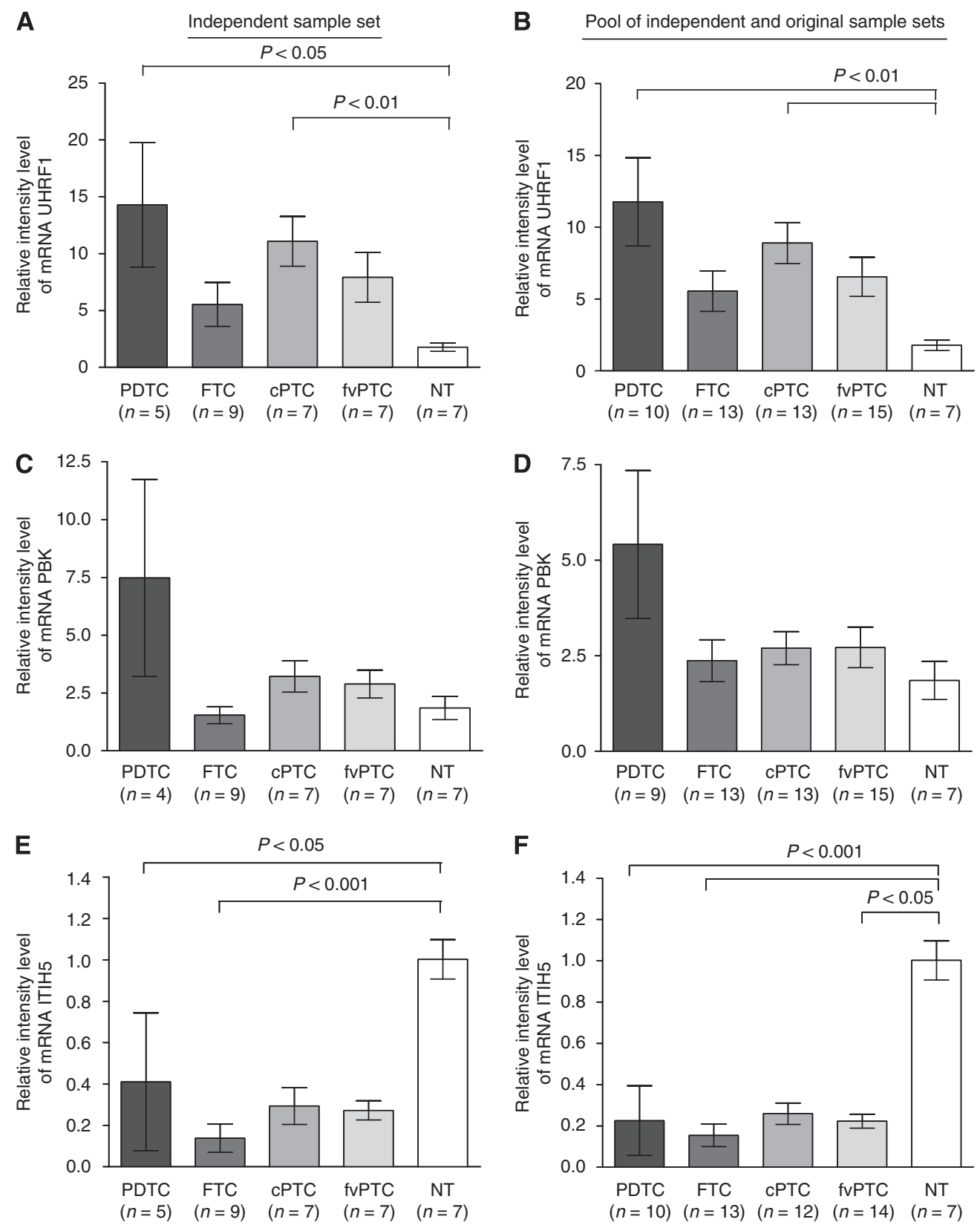

Figure 3 Expression of the UHRFI, PBK and ITIH5 genes in different tumour histotypes, assessed by quantitative RT-PCR. Relative mRNA levels for UHRFI (A, B), PBK (C, D) and ITIH5 (E, F) were assessed in an independent sample set (left panel) and in the entire sample set, comprising the microarray and the independent sample sets (right panel). Expression levels were normalised with the GAPDH expression and determined relatively to a calibrator. Error bars denote \pm s.e.m. The $P$ values for difference in mean expression between groups were performed using the Kruskal-Wallis with Dunn's Multiple Comparison test. These genes could not be assayed in one CPTC from the microarray set, PBK could not be evaluated in one PDTC sample, as well as, ITIH5 in one fvPTC and one CPTC from microarray set. CPTC = classic papillary thyroid carcinoma; FTC = follicular thyroid carcinoma; fvPTC = follicular variant of papillary thyroid carcinoma; PDTC = poorly differentiated thyroid carcinoma; NT = normal thyroid tissue.

\section{DISCUSSION}

The more aggressive thyroid carcinomas (PDTC and ATC) have high malignant potential and it is not yet clear whether they arise from pre-existing indolent WDTC or whether they arise de novo. Some PDTC cases bear areas of pre-existing PTC and have a significant prevalence of BRAF mutations (Nikiforova et al, 2003). Others, instead of BRAF, frequently display RAS mutations (GarciaRostan et al, 2003), which are typically detected in follicular thyroid adenomas, FTC and fvPTC. Comparative Genomic Hybridisation
(CGH) studies showed that among 11 copy number changes present in PTC, 8 were also present in the PDTC set, thus suggesting common genetic pathways (Wreesmann et al, 2002).

In our work, we were able to identify distinct gene expression profiles among different thyroid tumour histotypes. Our results suggest that PDTC have a gene expression profile closer to PTC, in particular to the follicular variant, than to FTC. In fact, for PDTC harbouring $R A S$ mutations, a clear similarity to the gene profile of $R A S$-mutated fvPTC was observed. Interestingly, these RASmutated PDTC, presented papillary like nuclei. In keeping with 
our findings, it has been observed that fvPTC, in contrast to cPTC, are more frequently aneuploid (Wreesmann et al, 2004), a feature common in PDTC. Therefore, fvPTC are likely to be precursors of PDTC, particularly those cases harbouring RAS mutations.

We also analysed the differential gene expression between tumours and normal thyroid tissues. PTC cases had slightly more over- than under-expressed probe sets, confirming previous reports (Huang et al, 2001). On the other hand, FTC and PDTC had clearly a predominance of downregulated probe sets, which is also in accordance with others (Aldred et al, 2003; Rodrigues et al, 2007). Studies have shown that allelic losses are clearly more frequent in FTC and PDTC than in PTC (Ward et al, 1998; Rodrigues et al, 2004). This could account for the differences in gene expression as a genomic loss could, theoretically, originate under-expression of genes. Epigenetic mechanisms, such as DNA hypermethylation, are also likely to explain these expression profiles. Indeed, increased frequency of hypermethylated $\mathrm{CpG}$ islands is a common alteration in tumour progression.

Eleven probe sets were simultaneously under-expressed in all tumours relatively to normal tissues, suggesting that these genes may have important suppressor activity in thyroid tumourigenesis. Among these, metallophosphoesterase domain containing 2 (MPPED2) and cellular retinoic acid-binding protein 1 (CRABP1) under-expression have already been observed in thyroid tumours (Griffith et al, 2006).

As observed earlier in other genome-wide studies, comparing clinically aggressive PTC with differentiated PTC cases (Fluge et al, 2006) or comparing PDTC with normal tissue (Rodrigues et al, 2007) or with WDTC (Montero-Conde et al, 2008), we found that many of the genes differentially upregulated in PDTC relatively to normal tissues were associated with the cell-cycle, indicating that the deregulation of this process is crucial in the progression to more aggressive thyroid tumours. In particular, we identified genes with major roles in mitosis, such as $\mathrm{CDC} 28$ protein kinase regulatory subunit $2(C K S 2)$ and cyclin E2 (CCNE2), which have been reported as over-expressed in various types of tumours (Gudas et al, 1999; Scrideli et al, 2008).

We selected three genes for real-time RT-PCR analysis, which were shown in the microarray analysis, to be differentially overexpressed ( $P B K$ and UHRF1) and under-expressed (ITIH5) between PDTC and normal thyroid tissues. UHRF1 and ITIH5 expressions were statistically different between PDTC and normal thyroid samples. Although not statistically significant, $P B K$ had higher expression levels in PDTC compared with normal thyroid. Therefore, $P B K$ is also a potential therapeutic target, as it encodes a mitotic protein, member of MAPK kinases family, which was found to be over-expressed in haematological (Nandi et al, 2004) and breast tumours (Park et al, 2006), and in PDTC cell lines (Rodrigues et al, 2007). UHRF1 over-expression has been already reported in lung (Jenkins et al, 2005) and breast (Hopfner et al, 2000) cancers. By conventional CGH analyses, the chromosomal locus 19p13.3, where UHRF1 is located, was identified as a common region of chromosomal gains in Hürthle cell thyroid neoplasms (Wada et al, 2002) and recently, using array-CGH, gains involving the 19p13 region were also found in $67 \%$ of ATC (Lee et al, 2008). UHRF1 encodes a nuclear protein that transcriptionally regulates TOP2A (Hopfner et al, 2000), an enzyme that catalyses the breaking and rejoining of DNA strands, during transcription. Interestingly, TOP $2 A$ showed a five-fold increase in PDTC $v s$ normal tissues $(P=0.02)$. In addition, UHRF1 is known to regulate the retinoblastoma protein (Jeanblanc et $a l, 2005)$ and is involved in the DNA damage response (Jenkins et al, 2005). More recently, an essential role for UHRF1 in the control of DNA methyltransferase 1 (DNMT1), the protein responsible for DNA methylation maintenance in mammalian cells, has also been reported (Bostick et al, 2007; Sharif et al, 2007). UHRF1 and DNMT1 interactions have been shown to be involved in VEGF regulation, a major pro-angiogenic protein (Achour et al, 2008).
Classification according to biological functions showed that about $8.5 \%$ of the genes differentially expressed between PDTC and normal tissues were downregulated and all related to cell adhesion. Interestingly, by further analysis, we found that underexpressed genes were mainly related to the cell membrane, encoding receptors, transmembranar or extracellular proteins. In agreement with this, the ITIH5 gene, which may have an essential function in cell attachment and invasion, was under-expressed in PDTC tumours. ITIH5 is a recently discovered member of the inter-alpha (globulin) inhibitor heavy chains (ITIH) gene family (Himmelfarb et al, 2004). In particular, the main function of ITIH is based on their covalent linkage to hyaluronic acid, the major component of the extracellular matrix. Therefore, deregulation of ITIH proteins affects the stability of the extracellular matrix and so, may promote tumour invasion and metastasis (Bost et al, 1998). Accordingly, ITIH genes have been shown to be downregulated in a variety of human tumours and have been proposed as tumour suppressor or metastasis repressor genes (Hamm et al, 2008). ITIH5 downregulation in breast cancer, caused by promoter hypermethylation, is associated with poorer clinical outcome, and reduced protein expression was proved to be a bad prognostic marker in invasive node-negative patients (Veeck et al, 2008). In fact, we found that lower expression of ITIH5 was statistically associated with larger tumours, as well as with more aggressive cases, such as PDTC (reaching undetectable levels in the two PDTC cell lines). Interestingly, we could also observe that extensively invasive FTC had lower ITIH5 expression levels than minimally invasive ones.

Compared with WDTC, PDTC had enriched gene sets (represented by over-expressed genes) associated earlier with cell cycle and poor prognosis signatures. Interestingly, one of these sets corresponded to a meta-signature of genes differentially overexpressed in undifferentiated relatively to well-differentiated cancers of different tissues (Rhodes et al, 2004). Among the most represented genes in these sets were cell-cycle regulators $(C D C 2$, $C C N B 2, C D K N 3$ and TOP2A), as well as genes with a role in the structure of the kinetochore and in the mitotic spindle assembly checkpoint (MSAC) (CENPA, BUB1 and MAD2L1). We also found statistically significant molecular signatures associated with the $B U B 1$ and $B U B 1 \beta$ (yeast) (BUB1B) genes. Some of these genes were reported earlier to be over-expressed in advanced cases of thyroid tumours (Montero-Conde et al, 2008; Wada et al, 2008). These observations indicate that PDTC may have abnormalities in MSAC or in the attachment of kinetochores, which may compromise mitotic fidelity and contribute to chromosomal instability. Accordingly, we observed earlier (Banito et al, 2007) that four out of the five PDTC analysed in this study were aneuploid.

In the analysis of genes differentially expressed between PDTC and WDTC, we only identified two genes. One of these, the 3-phosphoinositide-dependent protein kinase-1 gene (PDPK1), encodes a protein responsible for protein kinase $\mathrm{B}$ or AKT activation. PI3K/AKT pathway has a central role in regulation of apoptosis, proliferation and cell-cycle progression and its abnormal activation is frequently found in cancers, including thyroid tumours (Shinohara et al, 2007). Unexpectedly, and contrary to other cancer types, we observed that PDPK1 gene was underexpressed in PDTC.

The identification of molecular mechanisms involved in tumour progression is important in the design of new strategies for treating aggressive neoplasias, such as PDTC and ATC. For instance, over-expression of UHRF1 in PDTC samples, a protein that seems to be essential for DNMT1 function, indicates that UHRF1 targeting may offer a new therapeutic approach for PDTC cases. On the other hand, ITIH5 downregulation may be an essential mechanism in thyroid tumourigenesis, especially in tumour metastasis. In addition, and similarly to breast cancer, ITIH5 may prove to be a useful prognostic marker. 


\section{ACKNOWLEDGEMENTS}

We thank Dr Jörg D Becker, from the Affymetrix Core Facility at Instituto Gulbenkian de Ciência (Oeiras, Portugal) for the processing of RNA samples with $H G-U 133$ Plus 2.0 arrays. We also thank Drs Jorge Rosa Santos and Olimpia Cid for the surgical collection of tissue samples and Dr Rafael Cabrera for the histological analysis. Authors are also grateful to Pedro Batista for helpful assistance. This work was supported by Fundação Calouste Gulbenkian.

Supplementary Information accompanies the paper on British Journal of Cancer website (http://www.nature.com/bjc)

\section{REFERENCES}

Achour M, Jacq X, Rondé P, Alhosin M, Charlot C, Chataigneau T, Jeanblanc M Macaluso M, Giordano A, Hughes AD, Schini-Kerth VB, Bronner C (2008) The interaction of the SRA domain of ICBP90 with a novel domain of DNMT1 is involved in the regulation of VEGF gene expression. Oncogene 27: 2187-2197

Aldred MA, Ginn-Pease ME, Morrison CD, Popkie AP, Gimm O, Hoang-Vu C, Krause U, Dralle H, Jhiang SM, Plass C, Eng C (2003) Caveolin-1 and caveolin-2, together with three bone morphogenetic protein-related genes, may encode novel tumor suppressors down-regulated in sporadic follicular thyroid carcinogenesis. Cancer Res 63: 2864-2871

Banito A, Pinto AE, Espadinha C, Marques AR, Leite V (2007) Aneuploidy and RAS mutations are mutually exclusive events in the development of well-differentiated thyroid follicular tumors. Clin Endocrinol (Oxf) 67: $706-711$

Bost F, Diarra-Mehrpour M, Martin JP (1998) Inter-alpha-trypsin inhibitor proteoglycan family-a group of proteins binding and stabilizing the extracellular matrix. Eur J Biochem 252: 339-346

Bostick M, Kim JK, Estève PO, Clark A, Pradhan S, Jacobsen SE (2007) UHRF1 plays a role in maintaining DNA methylation in mammalian cells. Science 317: $1760-1764$

Ciampi R, Giordano TJ, Wikenheiser-Brokamp K, Koenig RJ, Nikiforov YE (2007) HOOK3-RET: a novel type of RET/PTC rearrangement in papillary thyroid carcinoma. Endocr Relat Cancer 14: 445-452

Dairkee SH, Ji Y, Ben Y, Moore DH, Meng Z, Jeffrey SS (2004) A molecular 'signature' of primary breast cancer cultures; patterns resembling tumor tissue. BMC Genomics 5: 47, doi:10.1186/1471-2164-5-47

DeLellis RA, Lloyd RV, Heitz PU, Eng C (eds) (2004) World Health Organization Classification of Tumors. Pathology and Genetics of Tumors of Endocrine Organs pp 50-83. IARC Press: Lyon, France

Eszlinger M, Krohn K, Kukulska A, Jarzab B, Paschke R (2007) Perspectives and limitations of microarray-based gene expression profiling of thyroid tumors. Endocr Rev 28: $322-338$

Fluge $\varnothing$, Bruland O, Akslen LA, Lillehaug JR, Varhaug JE (2006) Gene expression in poorly differentiated papillary thyroid carcinomas. Thyroid 16: $161-175$

Garcia-Rostan G, Zhao H, Camp RL, Pollan M, Herrero A, Pardo J, Wu R, Carcangiu ML, Costa J, Tallini G (2003) ras mutations are associated with aggressive tumor phenotypes and poor prognosis in thyroid cancer. J Clin Oncol 21: $3226-3235$

Griffith OL, Melck A, Jones SJ, Wiseman SM (2006) Meta-analysis and meta-review of thyroid cancer gene expression profiling studies identifies important diagnostic biomarkers. J Clin Oncol 24: 5043-5051

Gudas JM, Payton M, Thukral S, Chen E, Bass M, Robinson MO, Coats S (1999) Cyclin E2, a novel G1 cyclin that binds Cdk2 and is aberrantly expressed in human cancers. Mol Cell Biol 19: 612-622

Hamm A, Veeck J, Bektas N, Wild PJ, Hartmann A, Heindrichs U, Kristiansen G, Werbowetski-Ogilvie T, Del Maestro R, Knuechel R, Dahl E (2008) Frequent expression loss of Inter-alpha-trypsin inhibitor heavy chain (ITIH) genes in multiple human solid tumours: a systematic expression analysis. BMC Cancer 28: 8-25

Himmelfarb M, Klopocki E, Grube S, Staub E, Klaman I, Hinzmann B, Kristiansen G, Rosenthal A, Dürst M, Dahl E (2004) ITIH5, a novel member of the inter-alpha-trypsin inhibitor heavy chain family is downregulated in breast cancer. Cancer Lett 204: 69-77

Hopfner R, Mousli M, Jeltsch JM, Voulgaris A, Lutz Y, Marin C, Bellocq JP, Oudet P, Bronner C (2000) ICBP90, a novel human CCAAT binding protein, involved in the regulation of topoisomerase IIalpha expression. Cancer Res 60: $121-128$

Huang Y, Prasad M, Lemon WJ, Hampel H, Wright FA, Kornacker K, LiVolsi V, Frankel W, Kloos RT, Eng C, Pellegata NS, de la Chapelle A (2001) Gene expression in papillary thyroid carcinoma reveals highly consistent profiles. Proc Natl Acad Sci USA 98: 15044-15049
Irizarry RA, Hobbs B, Collin F, Beazer-Barclay YD, Antonellis KJ, Scherf U, Speed TP (2003) Exploration, normalization, and summaries of high density oligonucleotide array probe level data. Biostatistics 4: 249-264

Jeanblanc M, Mousli M, Hopfner R, Bathami K, Martinet N, Abbady AQ, Siffert JC, Mathieu E, Muller CD, Bronner C (2005) The retinoblastoma gene and its product are targeted by ICBP90: a key mechanism in the G1/S transition during the cell cycle. Oncogene 24: 7337-7345

Jenkins Y, Markovtsov V, Lang W, Sharma P, Pearsall D, Warner J, Franci C, Huang B, Huang J, Yam GC, Vistan JP, Pali E, Vialard J, Janicot M, Lorens JB, Payan DG, Hitoshi Y (2005) Critical role of the ubiquitin ligase activity of UHRF1, a nuclear RING finger protein, in tumor cell growth. Mol Biol Cell 16: 5621-5629

Khatri P, Draghici S, Ostermeier GC, Krawetz SA (2002) Profiling gene expression using onto-express. Genomics 79: 266-270

Lam KY, Lo CY, Chan KW, Wan KY (2000) Insular and anaplastic carcinoma of the thyroid: a 45-year comparative study at a single institution and a review of the significance of p53 and p21. Ann Surg 231: $329-338$

Lee JJ, Au AY, Foukakis T, Barbaro M, Kiss N, Clifton-Bligh R, Staaf J, Borg A, Delbridge L, Robinson BG, Wallin G, Höög A, Larsson C (2008) ArrayCGH identifies cyclin D1 and UBCH10 amplicons in anaplastic thyroid carcinoma. Endocr Relat Cancer 15: 801 -815

Li C, Wong WH (2001) Model-based analysis of oligonucleotide arrays: expression index computation and outlier detection. Proc Natl Acad Sci USA 98: $13144-13149$

Marques AR, Espadinha C, Catarino AL, Moniz S, Pereira T, Sobrinho LG, Leite V (2002) Expression of PAX8-PPAR gamma 1 rearrangements in both follicular thyroid carcinomas and adenomas. J Clin Endocrinol Metab 87: 3947 - 3952

Montero-Conde C, Martín-Campos JM, Lerma E, Gimenez G, Martínez-Guitarte JL, Combalía N, Montaner D, Matías-Guiu X, Dopazo J, de Leiva A, Robledo M, Maurício D (2008) Molecular profiling related to poor prognosis in thyroid carcinoma. Combining gene expression data and biological information. Oncogene 27: 1554-1561

Nandi A, Tidwell M, Karp J, Rapoport AP (2004) Protein expression of PDZ-binding kinase is up-regulated in hematologic malignancies and strongly down-regulated during terminal differentiation of HL-60 leukemic cells. Blood Cells Mol Dis 32: 240-245

Nikiforova MN, Kimura ET, Gandhi M, Biddinger PW, Knauf JA, Basolo F, Zhu Z, Giannini R, Salvatore G, Fusco A, Santoro M, Fagin JA, Nikiforov YE (2003) BRAF mutations in thyroid tumors are restricted to papillary carcinomas and anaplastic or poorly differentiated carcinomas arising from papillary carcinomas. J Clin Endocrinol Metab 88: $5399-5404$

Park JH, Lin ML, Nishidate T, Nakamura Y, Katagiri T (2006) PDZ-binding kinase/T-LAK cell-originated protein kinase, a putative cancer/testis antigen with an oncogenic activity in breast cancer. Cancer Res 66: 9186-9195

Patel NK, Shaha AR (2006) Poorly differentiated and anaplastic thyroid cancer. Cancer Control 13: 119-128

Pierotti MA, Greco A (2006) Oncogenic rearrangements of the NTRK1/NGF receptor. Cancer Lett 232: 90 -98

Quackenbush J (2006) Microarray analysis and tumor classification. N Engl J Med 354: $2463-2472$

Quiros RM, Ding HG, Gattuso P, Prinz RA, Xu X (2005) Evidence that one subset of anaplastic thyroid carcinomas are derived from papillary carcinomas due to BRAF and p53 mutations. Cancer 103: $2261-2268$

Rebelo S, Domingues R, Catarino AL, Mendonça E, Santos JR, Sobrinho L, Bugalho MJ (2003) Immunostaining and RT-PCR: different approaches to search for RET rearrangements in patients with papillary thyroid carcinoma. Int J Oncol 23: $1025-1032$ 
Rhodes DR, Yu J, Shanker K, Deshpande N, Varambally R, Ghosh D, Barrette T, Pandey A, Chinnaiyan AM (2004) Large-scale meta-analysis of cancer microarray data identifies common transcriptional profiles of neoplastic transformation and progression. Proc Natl Acad Sci USA 101: 9309-9314

Rodrigues RF, Roque L, Rosa-Santos J, Cid O, Soares J (2004) Chromosomal imbalances associated with anaplastic transformation of follicular thyroid carcinomas. Br J Cancer 90: 492-496

Rodrigues RF, Roque L, Krug T, Leite V (2007) Poorly differentiated and anaplastic thyroid carcinomas: chromosomal and oligo-array profile of five new cell lines. $\mathrm{Br} J$ Cancer 96: $1237-1245$

Scrideli CA, Carlotti Jr CG, Okamoto OK, Andrade VS, Cortez MA, Motta FJ, Lucio-Eterovic AK, Neder L, Rosemberg S, Oba-Shinjo SM, Marie SK, Tone LG (2008) Gene expression profile analysis of primary glioblastomas and non-neoplastic brain tissue: identification of potential target genes by oligonucleotide microarray and real-time quantitative PCR. J Neurooncol 88: $281-291$

Sharif J, Muto M, Takebayashi S, Suetake I, Iwamatsu A, Endo TA, Shinga J, Mizutani-Koseki Y, Toyoda T, Okamura K, Tajima S, Mitsuya K, Okano M, Koseki H (2007) The SRA protein Np95 mediates epigenetic inheritance by recruiting Dnmtl to methylated DNA. Nature 450: $908-912$

Shinohara M, Chung YJ, Saji M, Ringel MD (2007) AKT in thyroid tumorigenesis and progression. Endocrinology 148: $942-947$

Subramanian A, Tamayo P, Mootha VK, Mukherjee S, Ebert BL, Gillette MA, Paulovich A, Pomeroy SL, Golub TR, Lander ES, Mesirov JP (2005) Gene set enrichment analysis: a knowledge-based approach for interpreting genome-wide expression profiles. Proc Natl Acad Sci USA 102: $15545-15550$
Veeck J, Chorovicer M, Naami A, Breuer E, Zafrakas M, Bektas N, Dürst M, Kristiansen G, Wild PJ, Hartmann A, Knuechel R, Dahl E (2008) The extracellular matrix protein ITIH5 is a novel prognostic marker in invasive node-negative breast cancer and its aberrant expression is caused by promoter hypermethylation. Oncogene 27: 865-876

Wada N, Duh QY, Miura D, Brunaud L, Wong MG, Clark OH (2002) Chromosomal aberrations by comparative genomic hybridization in hürthle cell thyroid carcinomas are associated with tumor recurrence. J Clin Endocrinol Metab 87: 4595-4601

Wada N, Yoshida A, Miyagi Y, Yamamoto T, Nakayama H, Suganuma N, Matsuzu K, Masudo K, Hirakawa S, Rino Y, Masuda M, Imada T (2008) Overexpression of the mitotic spindle assembly checkpoint genes hBUB1, hBUBR1 and hMAD2 in thyroid carcinomas with aggressive nature. Anticancer Res 28: 139-144

Wang HM, Huang YW, Huang JS, Wang CH, Kok VC, Hung CM, Chen HM, Tzen CY (2007) Anaplastic carcinoma of the thyroid arising more often from follicular carcinoma than papillary carcinoma. Ann Surg Oncol 14: $3011-3018$

Ward LS, Brenta G, Medvedovic M, Fagin JA (1998) Studies of allelic loss in thyroid tumors reveal major differences in chromosomal instability between papillary and follicular carcinomas. J Clin Endocrinol Metab 83: $525-530$

Wreesmann VB, Ghossein RA, Hezel M, Banerjee D, Shaha AR, Tuttle RM, Shah JP, Rao PH, Singh B (2004) Follicular variant of papillary thyroid carcinoma: genome-wide appraisal of a controversial entity. Genes Chromosomes Cancer 40: 355-364

Wreesmann VB, Ghossein RA, Patel SG, Harris CP, Schnaser EA, Shaha AR, Tuttle RM, Shah JP, Rao PH, Singh B (2002) Genome-wide appraisal of thyroid cancer progression. Am J Pathol 161: 1549-1556 